\title{
Quality Control of Gamma Camera with SPECT Systems
}

\author{
Md. Razibul Hasan ${ }^{*}$, Md. Harunor Rashid Khan1, Md. Rashedur Rahman', Md. Shohel Parvez1, \\ Md. Rakibul Islam¹, Ashoke Kumar Paul²
}

\author{
${ }^{1}$ Physics Discipline, Khulna University, Khulna, Bangladesh \\ ${ }^{2}$ Institute of Nuclear Medicine \& Allied Sciences, Khulna, Bangladesh \\ Email: *razibreon@gmail.com
}

How to cite this paper: Hasan, Md.R., Khan, Md.H.R., Rahman, Md.R., Parvez, Md.S., Islam, Md.R. and Paul, A.K. (2017) Quality Control of Gamma Camera with SPECT Systems. International Journal of Medical Physics, Clinical Engineering and Radiation Oncology, 6, 225-232.

https://doi.org/10.4236/ijmpcero.2017.63021

Received: March 1, 2017

Accepted: June 18, 2017

Published: June 21, 2017

Copyright $\odot 2017$ by authors and Scientific Research Publishing Inc. This work is licensed under the Creative Commons Attribution International License (CC BY 4.0).

http://creativecommons.org/licenses/by/4.0/

\begin{abstract}
Quality control of Gamma Camera with SPECT System is highly valuable for assurance performance characteristic. We report the performance characteristic of gamma camera by intrinsic calibration and verification measurement. The study has been done using the data from Siemens Symbia S Series gamma camera by using a point source ${ }^{99 \mathrm{~m}} \mathrm{Tc}$ at the Institute of Nuclear Medicine \& Allied Sciences (INMAS), Khulna, Bangladesh. From intrinsic calibration and verification flood series, the integral uniformity for the central field of view (CFOV) has been found in between $4.01 \%$ and $2.88 \%$ and for the useful field of view (UFOV) has been in between $4.77 \%$ and $4.30 \%$. The differential uniformity for the CFOV has been in between $1.53 \%$ and $2.04 \%$ and for the UFOV has been in between $2.32 \%$ and $2.77 \%$. According to Operating Instruction Symbia System S Series manual, uniformity can compensate for values exceeding $10 \%$, however while integral uniformity exceed $7 \%$, have to contract Siemens customer service representative. In conclusion, these results show that the intrinsic uniformity of the gamma camera under this condition is within an acceptable range; thus the gamma camera working in INMAS is performed well.
\end{abstract}

\section{Keywords}

Gamma Camera, SPECT, Quality Control, Intrinsic Uniformity

\section{Introduction}

Gamma Camera is a major imaging device used in Nuclear Medicine. It is a diagnostic instrument which is used to image the radiation from a radiotracer inserted into patient's body. It scans the radiation area of the radiotracer and produces an image. The main purpose of Gamma Camera is to identify cancer tis- 
sues, proper abnormalities and other internal problems inside a patient's body. In the 1950s, Hal Anger conducted studies on medical imaging and from 1952 to 1958; he gradually developed the scintillation camera, also known as the Anger camera [1]. After developing gamma camera we get multiple gamma camera which generate a three dimensional image. Single photon emission computed tomography (SPECT) and positron emission tomography (PET) obeys this technology. In SPECT system, we get a scintillation camera mounted around the patient's body and it is connected to a proper computer system. The basic principle of a SPECT system dependent on the rotating camera concept is that a series of planar images are collected while the camera is rotated through either $180^{\circ}$ or $360^{\circ}$ around the patient [2]. The main purpose of Gamma camera with SPECT system is to scan brain heart, respiratory, liver and kidney. It is used for clinical studies producing better image and making diagnosis easier. The SPECT system is more sensitive than an ordinary gamma camera, so we should take a special technical support. The SPECT gamma camera is much more complex diagnostic instrument and it is more expensive to purchase and maintain.

The gamma camera is made up of many parts, each part performs as a specific function in converting gamma rays into light images and finally we get appropriate viewing image. The basic components of gamma camera are collimator, sodium iodide (NaI) crystal, photomultiplier tubes (PMT) and position logic circuit. Figure 1 illustrates the basic principles and components of the gamma camera.

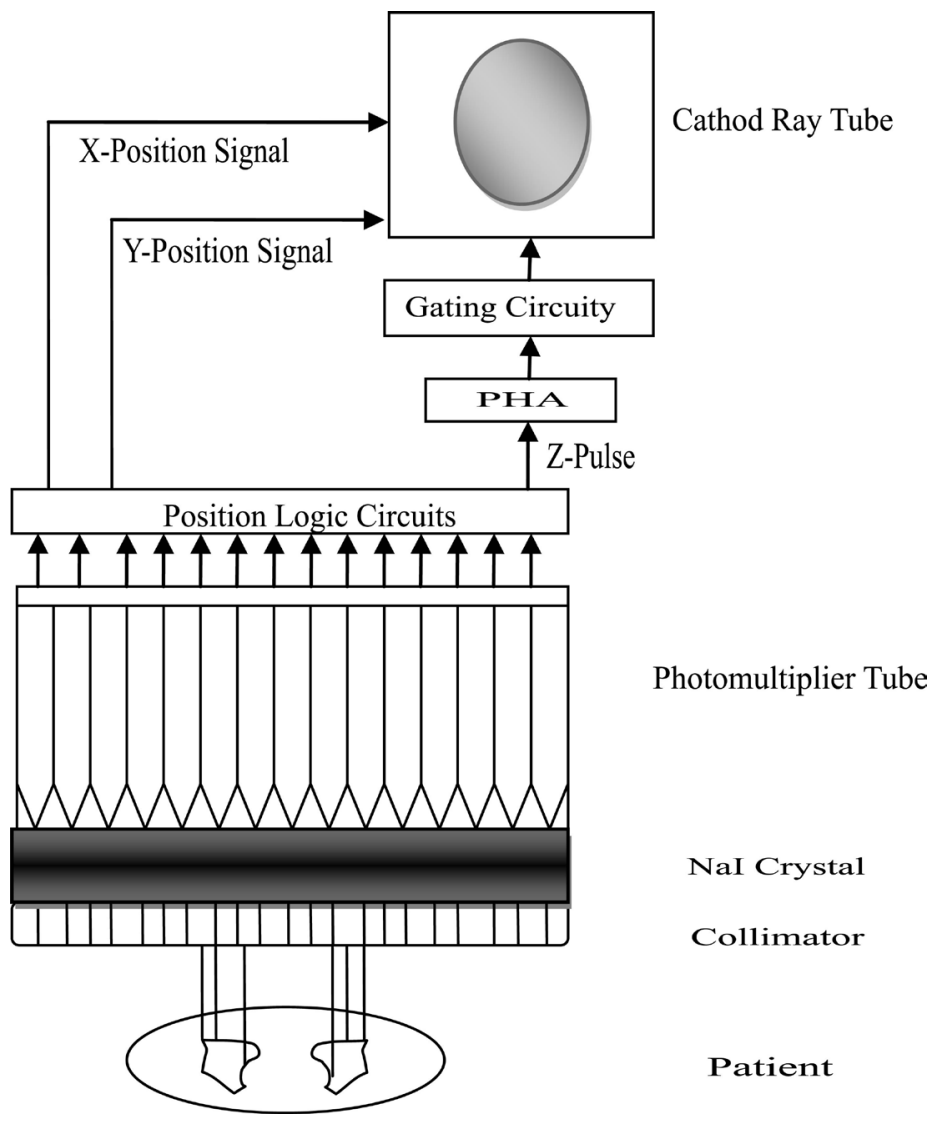

Figure 1. Basic principles and components of the gamma camera. 
The radiotracer emits gamma rays as it decays and the gamma rays travel towards the detector. The gamma rays have to pass through the collimators which ensure that it travels at a specified angle with respect to the detector crystal. The sodium iodide crystal converts the gamma rays into light. The photomultiplier tube converts the light into electrical signals. Finally these electrical signals are used to determine the position and the energy signals of the gamma rays.

A study of gamma camera intrinsic uniformity with SPECT system is presented as an index of quality assurance. Uniformity measurement is one of the parts of quality control of Gamma Camera. The uniformity measurements may be intrinsic or extrinsic. The intrinsic measurements are carried out without collimator and the extrinsic measurements are carried out with collimator [3]. It is one of the primary tests performed on the gamma cameras [4]. It is also one of the indices used to measure the performance of a gamma camera. Intrinsic flood uniformity test is a part of the quality assurance programme of a nuclear medicine department that ensures high standards of efficiency and reliability in the use of a gamma camera [5]. We thus avoid changes in the performance of a gamma camera system that might affect the interpretation of clinical studies [6]. Two uniformity parameters are computed for intrinsic uniformity measurement. These are integral uniformity and differential uniformity. Integral uniformity is a global parameter measuring uniformity over an extended area of the detector, expressed as follows [7]:

$$
\text { Integral uniformity }=\frac{(\operatorname{Max}-\operatorname{Min})}{(\operatorname{Max}+\operatorname{Min})} \times 100 \%
$$

Where Maxis the maximum count and Minis the minimum count found in any pixel within the specified area. Differential uniformity is a regional parameter that measures contrast over a small neighborhood. This measurement is performed using all $5 \times 1-$ pixel areas in both the $\mathrm{X}$ and $\mathrm{Y}$ directions, expressed as follows:

$$
\text { Differential uniformity }=\frac{\text { Largest deviation }(\text { Max }- \text { Min })}{(\operatorname{Max}+\operatorname{Min})} \times 100 \%
$$

The main aim of this study is to assure the performance characteristic of Gamma Camera by intrinsic calibration quality control studies (intrinsic calibration and verification measurement). This study was carried out on March 19, 2014.

\section{Materials and Methods}

From this study the following procedures was used for Siemens Symbia S Series Gamma Camera with double head (Siemens Medical Solutions U.S.A, Inc.). The Gamma Camera has two detectors which we indicated as Detector 1 and Detector 2. The specifications of the Gamma Camera are-thickness of the NaI crystal 9.5 or $15.9 \mathrm{~mm}$, size of the crystal $59.1 \times 44.5 \mathrm{~cm}$ (diagonal size $73.9 \mathrm{~cm}$ ), number of photo multiplier tube 59, the dimensions of the detector field of view (FOV) is $53.3 \times 38.7 \mathrm{~cm}$ (diagonal FOV is $65.9 \mathrm{~cm}$ ). ${ }^{99 \mathrm{~m}} \mathrm{Tc}$ gamma point source 


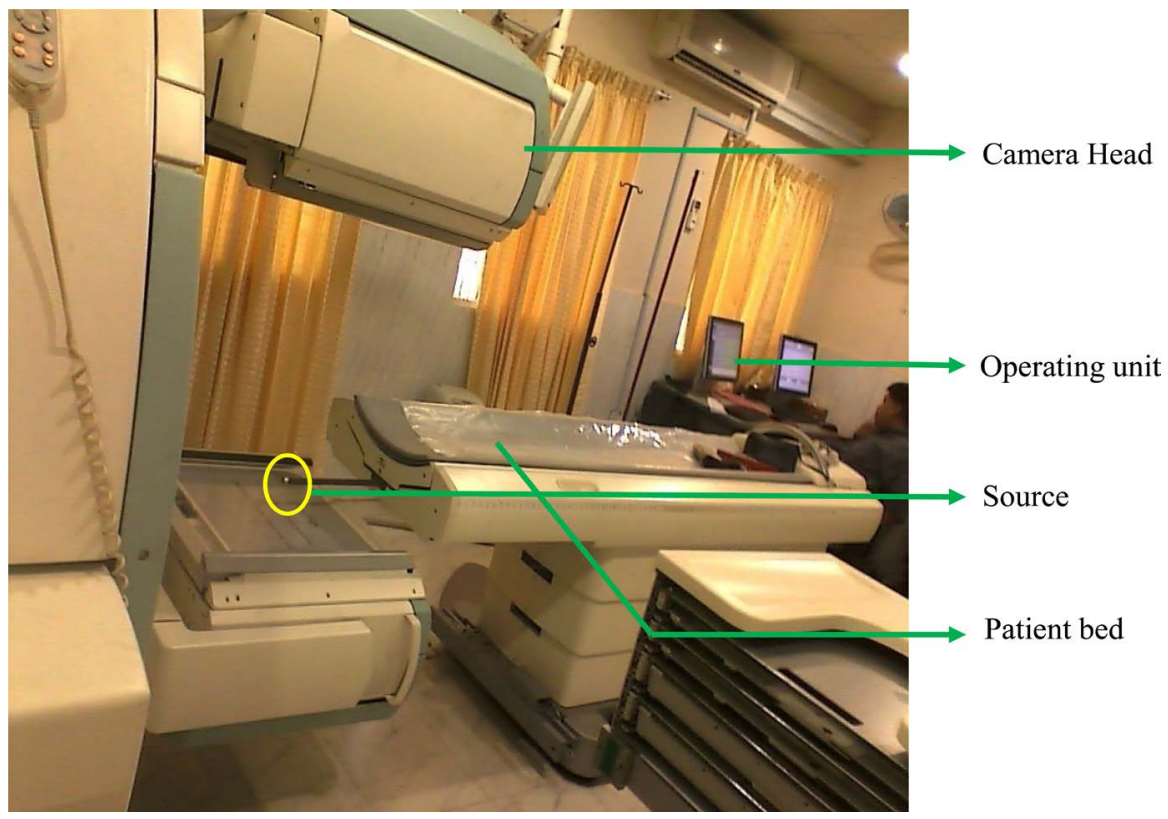

Figure 2. A partial view of gamma camera for this test.

activity, the number of acquired counts for the flood image, source-to-detector distance, image matrix size and source volume were evaluated to determine the ideal procedure for the quality control of Gamma Camera. Figure 2 shows a partial view of Gamma Camera for this test.

The collimator was removed from the camera. The integrated source holder was extended from its storage position on the rear bed and pulled until the source holder was approximately centered. The prepared point source in the vial was inserted, with the capped end of the vial, into the source holder. The activity of the point source was $28 \mu \mathrm{Ci}$ and its volume was $0.1 \mathrm{ml}$. The distance from point source-to-detector was at least 4 times the field of view (FOV) diameter from the detector. Camera surface and the room were cleaned to insure there was no contamination and verified that the background count rate display on the PPM (Patient Positioning Monitor) was less than $0.4 \mathrm{kcts} / \mathrm{sec}$. In performing intrinsic calibration and verification measurement checked that the count rate displayed on the PPM was between $15 \mathrm{kcts} / \mathrm{sec}$ and $50 \mathrm{kcts} / \mathrm{sec}$ to insure that the source strength was appropriate. In performing intrinsic calibration measurement the system acquired an uncorrected 200 million count flood for both detectors simultaneously and in performing intrinsic verification measurement the system acquired a 10 million count corrected flood on both detectors simultaneously. For both intrinsic calibration and verification measurement the image matrix size was $1024 \times 1024$ and a zoom factor was 1.00 and its adjusted energy window was $15 \%$. We initially followed the manufacture's instruction for the quality control test. All the working function of intrinsic calibration and verification measurement of the Gamma Camera (both differential \& integral uniformity) were performed using Singo and e.soft software provided by the manufacture. The integral and differential value was determined automatically by this SPECT Gamma Camera software. 


\section{Results and Discussion}

System uniformity is the most sensitive parameter to changes in system performance [8]. One of the things that affect image uniformity is the PMT (photo-multiplier tube) and detector's performance. Hence, intrinsic uniformity is probably the most important quality control (Q.C.) test that can be performed on a gamma camera system on a daily basis. One of the effects of PMT "drift" is the appearance of hot or cold spots in the flood image. This in a way shows that the uniformity of the images is dependent on the stability of the PMT and sometimes on the associated analogue electronic components. It is also dependent on the stability of the detector. When the uniformity of the camera is $\leq 2 \%$ the camera is considered to have a good uniformity [8].

In the present work the intrinsic uniformity of gamma camera installed at INMAS, Khulna, Bangladesh, was studied as an index of performance test. For this purpose intrinsic calibration flood series and verification measurement flood series were done which is shown in Figure 3 and Figure 4 respectively. Differential and integral uniformities for useful field of view (UFOV) and central field of view (CFOV) of Detector-1 and Detector-2 for intrinsic calibration and verification measurement were observed and the results are summarized in Table 1 and Table 2 respectively. The integral uniformity for the central field of view (CFOV) was found to be between $4.01 \%$ and $2.88 \%$ while the integral uniformity for the useful field of view (UFOV) was between $4.77 \%$ and $4.30 \%$. The differential uniformity for the CFOV was between $1.53 \%$ and $2.04 \%$ while that of the UFOV was between $2.32 \%$ and $2.77 \%$. The integral uniformity has typical values of $2 \%$ to $5 \%$ [9]. For differential uniformity in most cases, a value of less than $3 \%$ is obtained after uniformity correction [10]. When the value for

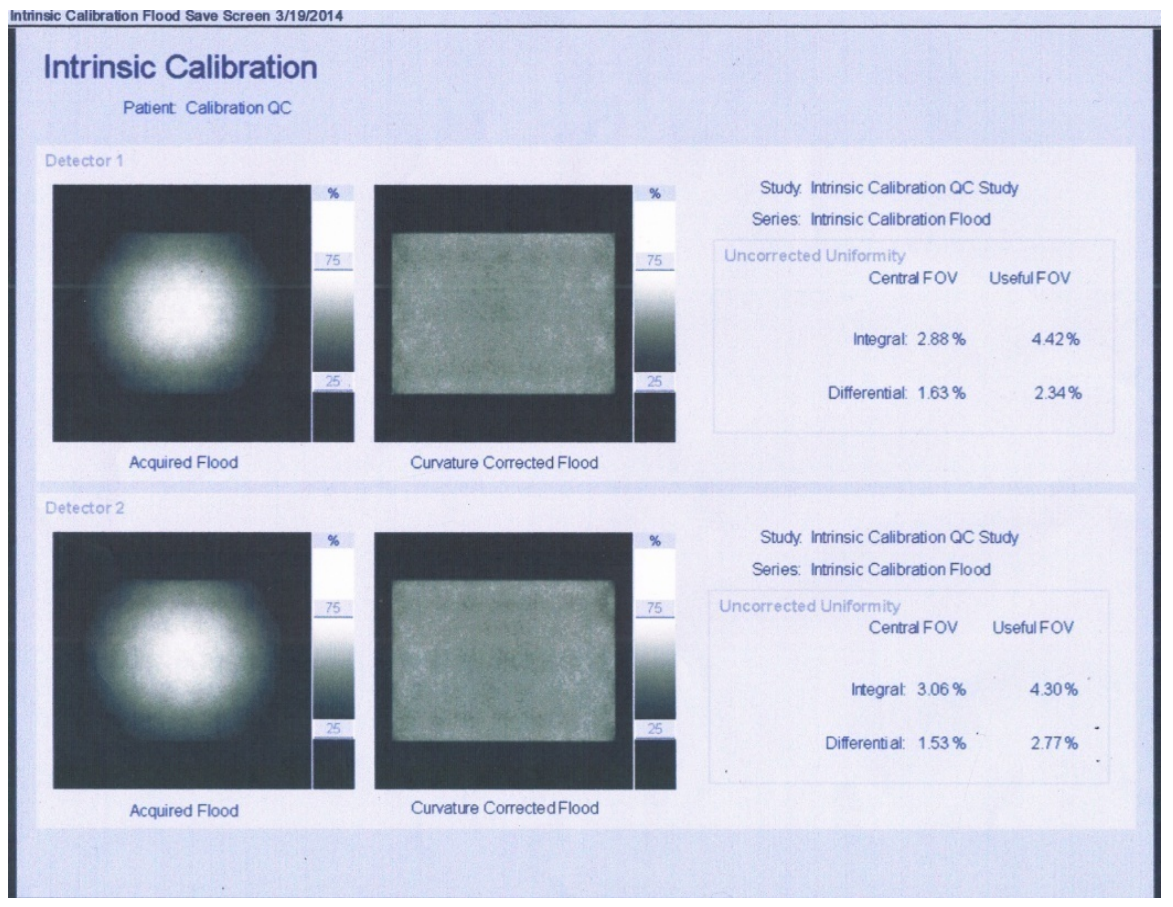

Figure 3. Intrinsic calibration flood series. 


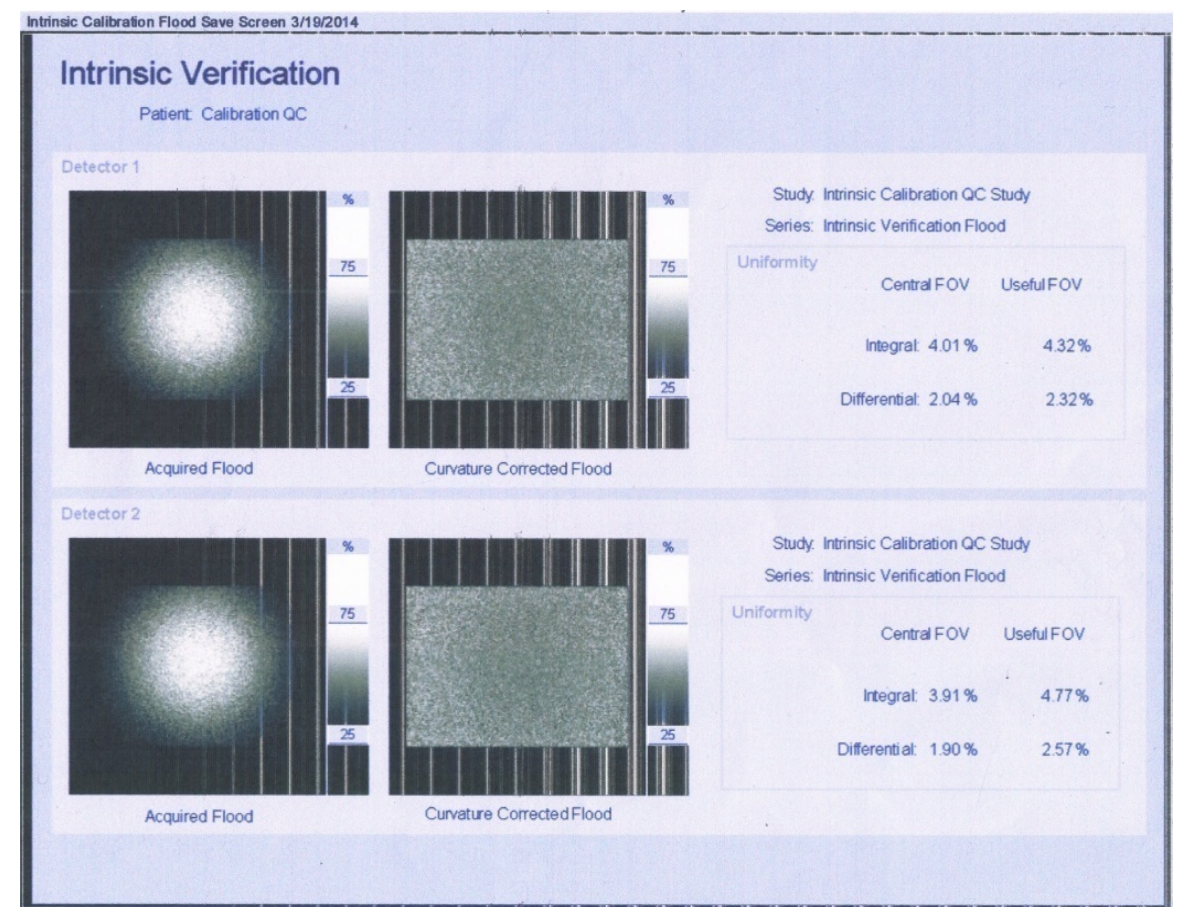

Figure 4. Intrinsic verification flood series.

Table 1. Intrinsic calibration measurement.

\begin{tabular}{lccc}
\hline \multirow{2}{*}{ Detector } & \multicolumn{2}{c}{ Intrinsic Uniformity (\%) } \\
\cline { 3 - 4 } Detector-1 & Differential Uniformity & UFOV & CFOV \\
& Integral Uniformity & 2.34 & 1.63 \\
& Differential Uniformity & 4.42 & 2.88 \\
Detector-2 & Integral Uniformity & 2.77 & 1.53 \\
& & 4.30 & 3.06 \\
\hline
\end{tabular}

Table 2. Intrinsic verification measurement.

\begin{tabular}{lccc}
\hline & & \multicolumn{2}{c}{ Intrinsic Uniformity (\%) } \\
\cline { 3 - 4 } Detector & UFOV & CFOV \\
\hline \multirow{2}{*}{ Detector-1 } & Differential Uniformity & 2.32 & 2.04 \\
& Integral Uniformity & 4.32 & 4.01 \\
\multirow{2}{*}{ Detector-2 } & Differential Uniformity & 2.57 & 1.09 \\
& Integral Uniformity & 4.77 & 3.91 \\
\hline
\end{tabular}

differential uniformity exceeds $3 \%$, maintenance service should be carried out on the gamma camera [11]. From these results, it was clear that the gamma camera had flood uniformity that was within an acceptable range.

For major uniformity defects consistent with crystal damage or detector malfunction, the intrinsic calibration flood should be reviewed. Mild variations in uniformity may occur with a properly functioning detector and will be removed by the uniformity correction. Uniformity values may be used as a guide to dis- 
tinguish between acceptable and unacceptable levels of uniformity. The intrinsic calibration concludes with a corrected verification flood to assess the corrected performance of the system immediately following calibration and the results of this flood may be used as a baseline for comparison of subsequent daily intrinsic verification floods. Provided the intrinsic calibration flood was performed correctly, the corrected intrinsic verification flood should appear visually uniform with the uniformity numbers determined by the statistics of the low count flood and the results of this flood may be used as a baseline for comparison of subsequent daily intrinsic verification floods [12].

\section{Conclusion}

Uniformity measurement is one of the parts of performance test of gamma camera. In this work the intrinsic uniformity of gamma camera with SPECT system was studied by intrinsic calibration and verification measurement flood series technique. From this study, we observed that differential and integral uniformities under this condition do not exceed the Operating Instruction Symbia System S Series Manual value (10\% for DU and 3\% for IU). Thus it may be concluded that the gamma camera working in INMAS at Khulna in Bangladesh is performed well.

\section{Acknowledgements}

The authors are highly grateful to all the staffs of the Institute of Nuclear Medicine \& Allied Sciences (INMAS), Khulna, Bangladesh for providing the necessary facilities during the work.

\section{References}

[1] TiP-TV ${ }^{\mathrm{sm}}$ Training in Partnership Program Supplement for Imaging Professionals (2004) NM/PET: Advances in Gamma Camera Technology.

[2] International Atomic Energy Agency (2009) Quality Assurance for SPECT Systems. IAEA Human Health Series No. 6. International Atomic Energy Agency, Vienna.

[3] Gar-Elnabi, M.E.M., Ali, W.M., Omer, M.A.A., Sam, A.K. and Edam, G.A. (2015) Development and Assessment of Quality Control Phantom for Linearity and Uniformity. Open Journal of Radiology, 5, 59-65. https://doi.org/10.4236/ojrad.2015.52010

[4] National Electrical Manufacturers Association (NEMA) (2001) Performance Measurements of Scintillation Cameras. Global Engineering Document. NEMA Standards Publication NU, Rosslyn, Virginia.

[5] Ejeh, J.E., Adedapo, K.S., Akinlade, B.I. and Osifo, B.O.A. (2011) Gamma Camera Intrinsic Uniformity in an Unstable Power Supply Environment. Hellenic Journal of Nuclear Medicine, 14, 146-148. http://nuclmed.web.auth.gr/magazine/eng/may11/7.pdf

[6] Graham, S.L. (1995) Quality Control for SPECT Systems, the AAPM/RSNA Physics Tutorials for Residents. Imaging and Therapeutic Technology, 15, 1471-1481.

[7] Nichols K.J., et al. (2007) ASNC Imaging Guidelines for Nuclear Cardiology Procedures. Instrumentation Quality Assurance and Performance. Journal of Nuclear Cardiology, 14, 61-78. https://doi.org/10.1016/j.nuclcard.2007.09.024 
[8] Graham, S.L., Levin, C.S. and Muehllehner, G. (2003) Anger Scintillation Camera. In: Sandler, M.P., Coleman, R.E., Patton, J.A., ThWackers, F.J. and Gottschalk, A., Eds., Diagnostic Nuclear Medicine, Lippincott Williams and Wilkins Philadelphia, Philadelphia, 31-42.

[9] Cherry, S.R., Sorenson, J.A. and Phelps, M.E. (2003) Physics in Nuclear Medicine. Elsevier Science, Philadelphia, 250-262.

[10] O’Connor, M.K. and Vermeersch, C. (1991) Critical Examination of the Uniformity Requirements for Single Photon Emission Computed Tomography. Medical Physics, 18, 190-197. https://doi.org/10.1118/1.596706

[11] Young, K.C., Kouris, K., Awdeh, M. and Abdel-Dayem, H.M. (1990) Reproducibility and Action Levels for Gamma Camera Uniformity. Nuclear Medicine Communications, 11, 95-101.

https://doi.org/10.1097/00006231-199002000-00003

[12] Syngo MI Applications (2009) Operating Instruction Symbia System S Series. https://www.usa.siemens.com/healthcare

Submit or recommend next manuscript to SCIRP and we will provide best service for you:

Accepting pre-submission inquiries through Email, Facebook, LinkedIn, Twitter, etc. A wide selection of journals (inclusive of 9 subjects, more than 200 journals)

Providing 24-hour high-quality service

User-friendly online submission system

Fair and swift peer-review system

Efficient typesetting and proofreading procedure

Display of the result of downloads and visits, as well as the number of cited articles

Maximum dissemination of your research work

Submit your manuscript at: http://papersubmission.scirp.org/

Or contact ijmpcero@scirp.org 